\title{
Quantifying the Unstable in Linearized Nonlinear Systems
}

\author{
Graziano Chesi \\ Department of Electrical and Electronic Engineering \\ The University of Hong Kong \\ http://www.eee.hku.hk/ chesi
}

\begin{abstract}
It has been shown that quantifying the unstable in linear systems is important for establishing the existence of stabilizing feedback controllers in the presence of communications constraints. In this context, the instability measure is defined as the sum of the real parts (continuous-time case) or the product of the magnitudes (discrete-time case) of the unstable eigenvalues. This paper addresses the problem of quantifying the unstable in linearized systems obtained from nonlinear systems for a family of constant inputs, i.e., quantifying the largest instability measure over all admissible equilibrium points and all admissible constant inputs. It is supposed that the dynamics of the nonlinear system is polynomial in both state and input, either continuous-time or discrete-time, and that the set of constant inputs is a semialgebraic set. Two cases are considered: first, when the equilibrium points are known polynomial functions of the input, and, second, when the equilibrium points are unknown (polynomial or nonpolynomial) functions of the input. It is shown that upper bounds of the sought instability measure can be established through linear matrix inequalities (LMIs) by searching for polynomially-dependent Lyapunov function candidates. Moreover, it is shown that these upper bounds are nonconservative for a sufficiently large degree of the Lyapunov function candidates under some conditions. Lastly, necessary and sufficient conditions are provided for establishing whether the obtained upper bounds are nonconservative. Some numerical examples also show the advantages of the proposed method with respect to grid techniques.
\end{abstract}

\section{INTRODUCTION}

An important issue in control systems consists of quantifying the unstable. Indeed, it has been shown that this allows one to establish the existence of stabilizing feedback controllers 
for linear systems in the presence of communications constraints. In this context, the instability measure is defined as the sum of the real parts (continuous-time case) or the product of the magnitudes (discrete-time case) of the unstable eigenvalues. For instance, [1] shows that the coarsest quantizer that quadratically stabilizes a single-input discrete-time system is logarithmic, and provides a closed form for the optimal logarithmic base in terms of this instability measure. Also, [2] considers the design of encoders, decoders, and controllers to achieve different control objectives, and provides upper and lower bounds on the required channel rate based on the instability measure. Analogous results are proposed in [3] where the lowest quantization density of infinite logarithmic quantizers in a single-input system is investigated, in [4] where the case of multiple sensors that partially observe the system is considered, in [5] where a virtual system approach for digital finite communication bandwidth control is described, and in [6] for the case of channel modeled by a finite logarithmic quantizer. The reader is also referred to [7]-[9] for further applications of the instability measure, and to [10], [11] for connections with the entropy of dynamical systems.

The linear system that has to be considered when quantifying the unstable is very often a linearized nonlinear system. Indeed, real plants are generally characterized by nonlinear dynamics. Moreover, considering the linearized system obtained for a certain equilibrium point and for a certain constant input is generally sufficiently for designing a locally stabilizing feedback controller. Unfortunately, the constant input is very often unknown, for instance because the user is allowed to change it in order to choose a desired performance. Consequently, the linearized system is unknown as well, and its dependence on the unknown constant input can be very complex since also the equilibrium point depends on the unknown constant input. In the context of uncertain linear systems, the instability measure has been investigated in [12] in the case of uncertain parameters constrained in a polytope by exploiting Lyapunov functions, and in [13] in the case of uncertain parameters constrained in a semialgebraic set by exploiting determinants.

This paper addresses the problem of quantifying the unstable in linearized systems obtained from nonlinear systems for a family of constant inputs, i.e., quantifying the largest instability measure over all admissible equilibrium points and all admissible constant inputs. It is supposed that the dynamics of the nonlinear system is polynomial in both state and input, either continuoustime or discrete-time, and that the set of constant inputs is a semialgebraic set. Two cases are considered: first, when the equilibrium points are known polynomial functions of the input, and, 
second, when the equilibrium points are unknown (polynomial or non-polynomial) functions of the input. It is shown that upper bounds of the sought instability measure can be established through linear matrix inequalities (LMIs) by searching for polynomially-dependent Lyapunov function candidates. Moreover, it is shown that these upper bounds are nonconservative for a sufficiently large degree of the Lyapunov function candidates under some conditions. Lastly, necessary and sufficient conditions are provided for establishing whether the obtained upper bounds are nonconservative.

Let us observe that a possible way to address this problem is via grid techniques. Specifically, one could generate samples of the set of admissible constant inputs and evaluate the instability measure for each of them. However, this technique suffers of several issues. The first issue is that, in spite of the number of samples used, only lower bounds of the largest instability measure can be obtained (since the set of admissible constant inputs is continuous), while upper bounds are needed in order to ensure stability. The second issue is that, for each sample, one should compute the set of admissible equilibrium points, which amounts to solving a system of polynomial equations. Unfortunately, solving a system of polynomial equations is a difficult task and no reliable method does exist for this. Indeed, symbolic methods like resultants suffer of the problem that the univariate polynomial generated can have huge degree, while numerical methods like homotopy methods suffer of the problem that solutions might be lost, see [14], [15] and references therein. The third issue is that the computational time can be very large depending on the the number of samples used. These issues are illustrated with some numerical examples in Section V.

The paper is organized as follows. Section II introduces the problem formulation. Section III describes the derivation of the upper bounds. Section IV presents the conservatism analysis and the extension to the case of discrete-time nonlinear systems. Section V presents some illustrative examples. Lastly, Section VI concludes the paper with some final remarks. A conference version of this paper (considering only continuous-time dynamics, and without the conservatism analysis) appeared as reported in [16].

\section{PRELIMINARIES}

The notation used throughout the paper is as follows: $\mathbb{R}$ : space of real numbers; $\mathbb{C}$ : space of complex numbers; $0_{n}: n \times 1$ null vector; $\mathbb{R}_{0}^{n}: \mathbb{R}^{n} \backslash\left\{0_{n}\right\} ; I$ : identity matrix (of size specified by 
the context); $A^{\prime}$ : conjugate transpose of matrix $A ; A>0, A \geq 0$ : hermitian positive definite and semidefinite matrix $A ; \Re(a), \Im(a)$ : real and imaginary parts of $a \in \mathbb{C} ;|a|$ : magnitude of $a \in \mathbb{C}$; $\operatorname{spec}(A)$ : spectrum of matrix $A ; \lambda_{\min }(A)$ : minimum real eigenvalue of matrix $A$.

Let us start by considering the continuous-time nonlinear system

$$
\dot{x}(t)=f(x(t), u(t))
$$

where $t \in \mathbb{R}$ is the time, $x \in \mathbb{R}^{n}$ is the state vector, $u \in \mathbb{R}^{m}$ is the input vector, and $f(x(t), u(t))$ is a polynomial function in $x(t)$ and $u(t)$.

Let $\phi \in \mathbb{R}^{m}$ be a reference value of interest of the input vector, and let $\Theta(\phi) \subseteq \mathbb{R}^{n}$ be the set of equilibrium points of the nonlinear system (1) corresponding to $\phi$, i.e.,

$$
\Theta(\phi)=\left\{\theta \in \mathbb{R}^{n}: f(\theta, \phi)=0\right\}
$$

Let $\theta \in \Theta(\phi)$ be an equilibrium point of interest of the nonlinear system (1) corresponding to $\phi$. For local stabilization in a neighbourhood of the pair $(\theta, \phi)$, the nonlinear system (1) is generally approximated with its linearized form

$$
\dot{\tilde{x}}(t)=A(\theta, \phi) \tilde{x}(t)+B(\theta, \phi) \tilde{u}(t)
$$

where

$$
\left\{\begin{array}{l}
\tilde{x}(t)=x(t)-\theta \\
\tilde{u}(t)=u(t)-\phi
\end{array}\right.
$$

are the variations of the state vector and input vector with respect to $\theta$ and $\phi$, respectively, and

$$
\left\{\begin{array}{l}
A(\theta, \phi)=\left.\frac{d f(x, u)}{d x}\right|_{(x, u)=(\theta, \phi)} \\
B(\theta, \phi)=\left.\frac{d f(x, u)}{d u}\right|_{(x, u)=(\theta, \phi)}
\end{array}\right.
$$

are the matrices that describe the local behaviour of the system.

In the literature, several conditions for the existence of a stabilizing controller for the linearized system (3) under communications constraints have been given based on a certain instability measure of the matrix $A(\theta, \phi)$, which has to be smaller than a specific value depending on the 
communications constraints considered. Specifically, let $M \in \mathbb{R}^{n \times n}$. In the continuous-time case, such an instability measure is defined as

$$
\mu(M)=\sum_{i=1}^{n} \max \left\{0, \Re\left(\lambda_{i}(M)\right)\right\}
$$

where $\lambda_{i}(M)$ is the $i$-th eigenvalue of $M$.

If the reference value $\phi$ of the input vector and the equilibrium point $\theta$ of the nonlinear system (1) are known, then the instability measure to consider is known as well, since the matrix $A(\theta, \phi)$ is a constant for such values of $\theta$ and $\phi$.

However, the pair $(\theta, \phi)$ is very often unknown due to the following reasons:

1) the reference value $\phi$ might change, for instance as the result of a choice of the user;

2) the equilibrium point $\theta$ might be uncertain in the set $\Theta(\phi)$, for instance due to the initial condition of the nonlinear system (1).

Due to this uncertainty on the pair $(\theta, \phi)$, it appears clear that one should determine the largest value of the instability measure over all admissible pairs $(\theta, \phi)$ in order to ensure the existence of a stabilizing controller for the linearized system (3) under communications constraints.

Let us denote with $\Phi \subseteq \mathbb{R}^{m}$ the set of admissible reference values of the input vector, i.e.,

$$
\phi \in \Phi
$$

We suppose that $\Phi$ is a semialgebraic set expressed as

$$
\Phi=\left\{\phi \in \mathbb{R}^{m}: a_{i}(\phi) \geq 0, i=1, \ldots, n_{a}\right\}
$$

where $a_{i}(\phi), i=1, \ldots, n_{a}$, are polynomials. The first problem addressed in this paper is formulated as follows.

Problem 1. Determine upper bounds of the largest instability measure $\mu(A(\theta, \phi))$ over the admissible pairs $(\theta, \phi)$, i.e.,

$$
\mu^{*}=\sup _{\substack{\phi \in \Phi \\ \theta \in \Theta(\phi)}} \mu(A(\theta, \phi)) .
$$




\section{UPPER BOUNDS}

This section describes the method proposed in this paper for determining upper bounds of $\mu^{*}$ in (9). Let us start by introducing the following two cases.

1) Case I: for all $\phi \in \Phi$, the equilibrium points of the nonlinear system (1) can be expressed by polynomial functions of $\phi$. That is, there exist polynomial functions $g_{j}: \mathbb{R}^{m} \rightarrow \mathbb{R}^{n}$, $j=1, \ldots, n_{g}$, such that the set of equilibrium points $\Theta(\phi)$ in (2) can be written as

$$
\Theta(\phi)=\left\{g_{1}(\phi), \ldots, g_{n_{g}}(\phi)\right\} \quad \forall \phi \in \Phi .
$$

2) Case II: any possibility (including Case I).

The next two subsections will consider separately these cases.

\section{A. Case I}

In this section we suppose that (10) holds for some polynomial functions $g_{j}(\phi), j=1, \ldots, n_{g}$. The following example illustrates such a situation.

Example 1. Let us consider (1) with

$$
f(x, u)=\left(\begin{array}{c}
-2 x_{3}-x_{2}^{2}-2 x_{1} u_{2}^{2} \\
x_{2}-x_{1}^{2}+0.5 x_{1} u_{2} \\
x_{1}+x_{2}+x_{1} u_{1}
\end{array}\right) .
$$

The set $\Phi$ and its expression in (8) are chosen as

$$
\left\{\begin{aligned}
\Phi & =[-1,1]^{2} \\
a_{i}(\phi) & =1-\phi_{i}^{2} \quad \forall i=1,2 .
\end{aligned}\right.
$$

It follows that the set of equilibrium points $\Theta(\phi)$ in (2) can be written as in (10) for polynomial functions $g_{j}(\phi), j=1,2$, given by

$$
\left\{\begin{array}{c}
g_{1}(\phi)=\left(\begin{array}{l}
0 \\
0 \\
0
\end{array}\right), \quad g_{2}(\phi)=\left(\begin{array}{c}
\alpha(\phi) \\
\beta(\phi) \\
-\phi_{2}^{2} \alpha(\phi)-0.5 \beta(\phi)^{2}
\end{array}\right) . \\
\alpha(\phi)=-1-\phi_{1}+0.5 \phi_{2} \\
\beta(\phi)=-\left(1+\phi_{1}\right) \alpha(\phi) .
\end{array}\right.
$$


In order to determine $\mu^{*}$ in (9) in this case, let us proceed as follows. For $k \in\{1, \ldots, n\}$ define the binomial coefficient

$$
c_{k}=\frac{n !}{(n-k) ! k !}
$$

Let $M \in \mathbb{R}^{n \times n}$, and let $\Omega_{k}(M)$ be a $c_{k} \times c_{k}$ matrix function satisfying

$$
\operatorname{spec}\left(\Omega_{k}(M)\right)=\left\{\sum_{i=1}^{k} \lambda_{z_{i}}(M), z \in \mathcal{I}_{k}\right\}
$$

where $M \in \mathbb{R}^{n \times n}$ and $\mathcal{I}_{k}$ is the set of $k$-tuples in $\{1, \ldots, n\}$, i.e.,

$$
\begin{aligned}
\mathcal{I}_{k}= & \left\{\left(z_{1}, \ldots, z_{k}\right): z_{i} \in\{1, \ldots, n\}, z_{i}<z_{i+1}\right. \\
& \forall i=1, \ldots, k-1\} .
\end{aligned}
$$

A matrix function $\Omega_{k}(M)$ satisfying (15) can be directly built from $M$ following the idea described in [17]. For instance, for $n=3$ one has

$$
\left\{\begin{array}{l}
\mathcal{I}_{1}=\{z(1)=1, z(2)=2, z(3)=3\} \\
\mathcal{I}_{2}=\{z(1)=(1,2), z(2)=(1,3), z(3)=(2,3)\} \\
\mathcal{I}_{3}=\{z(1)=(1,2,3)\}
\end{array}\right.
$$

and

$$
\left\{\begin{aligned}
\Omega_{1}(M) & =M \\
\Omega_{2}(M) & =\left(\begin{array}{ccc}
M_{1,1}+M_{2,2} & M_{2,3} & -M_{1,3} \\
M_{3,2} & M_{1,1}+M_{3,3} & M_{1,2} \\
-M_{3,1} & M_{2,1} & M_{2,2}+M_{3,3}
\end{array}\right) \\
\Omega_{3}(M) & =\operatorname{trace}(M) .
\end{aligned}\right.
$$

The matrix function $\Omega_{k}(M)$ can be exploited as follows. Let us define

$$
w_{\min }=0
$$

and $\sigma:\left(w_{\text {min }}, \infty\right) \times \mathbb{C} \rightarrow \mathbb{R}$ as

$$
\sigma(w, \lambda)=w-\Re(\lambda)
$$

For $j \in\left\{1, \ldots, n_{g}\right\}, k \in\{1, \ldots, n\}$ and $w \in\left(w_{\min }, \infty\right)$, let us introduce

$$
D_{j, k}(\phi)=\Omega_{k}\left(A\left(g_{j}(\phi), \phi\right)\right)-w I
$$


which is a matrix polynomial since $g_{j}(\phi)$ is polynomial, $A(\theta, \phi)$ is polynomial, and $\Omega_{k}(M)$ is linear. Moreover, for a symmetric matrix polynomial $P_{j, k}: \mathbb{R}^{m} \rightarrow \mathbb{R}^{c_{k} \times c_{k}}$, let us define

$$
Q_{j, k}(\phi)=-P_{j, k}(\phi) D_{j, k}(\phi)-D_{j, k}(\phi)^{\prime} P_{j, k}(\phi)
$$

Lastly, let us introduce the following definition: a symmetric matrix polynomial $H: \mathbb{R}^{m} \rightarrow$ $\mathbb{R}^{h \times h}$ is said to be sum of squares (SOS) if there exist matrix polynomials $H_{i}: \mathbb{R}^{m} \rightarrow \mathbb{R}^{h \times h}$, $i=1, \ldots, i_{\max }$, such that

$$
H(\phi)=\sum_{i=1}^{i_{\max }} H_{i}(\phi)^{\prime} H_{i}(\phi) .
$$

The following result provides a condition for establishing an upper bound of $\mu^{*}$ based on a convex optimization problem.

Theorem 1: Let $w \in\left(w_{\text {min }}, \infty\right)$. Let us suppose that there exist $\varepsilon>0$ and symmetric matrix polynomials $P_{j, k}, R_{i, j, k, l}: \mathbb{R}^{m} \rightarrow \mathbb{R}^{c_{k} \times c_{k}}, i \in\left\{1, \ldots, n_{a}\right\}, j \in\left\{1, \ldots, n_{g}\right\}, k \in\{1, \ldots, n\}$ and $l \in\{1,2\}$, such that

$$
\left.\begin{array}{l}
P_{j, k}(\phi)-S_{j, k, 1}(\phi)-I \\
Q_{j, k}(\phi)-S_{j, k, 2}(\phi)-\varepsilon I \\
R_{i, j, k, l}(\phi)
\end{array}\right\} \text { are SOS }
$$

for all $i \in\left\{1, \ldots, n_{a}\right\}, j \in\left\{1, \ldots, n_{g}\right\}, k \in\{1, \ldots, n\}$ and $l \in\{1,2\}$, where

$$
S_{j, k, l}(\phi)=\sum_{i=1}^{n_{a}} a_{i}(\phi) R_{i, j, k, l}(\phi) .
$$

Then,

$$
\mu^{*}<w
$$

Proof. Suppose that (22) holds. Let $i \in\left\{1, \ldots, n_{a}\right\}, j \in\left\{1, \ldots, n_{g}\right\}, k \in\{1, \ldots, n\}, l \in\{1,2\}$ and $\phi \in \mathbb{R}^{m}$. From (21) it follows that

$$
\left\{\begin{aligned}
P_{j, k}(\phi)-S_{j, k, 1}(\phi)-I & \geq 0 \\
Q_{j, k}(\phi)-S_{j, k, 2}(\phi)-\varepsilon I & \geq 0 \\
R_{i, j, k, l}(\phi) & \geq 0 .
\end{aligned}\right.
$$


Let $\phi \in \Phi$. Since $R_{i, j, k, l}(\phi) \geq 0$ and $a_{i}(\phi) \geq 0$, it follows that

$$
\begin{aligned}
I & \leq P_{j, k}(\phi)-S_{j, k, 1}(\phi) \\
& =P_{j, k}(\phi)-\sum_{i=1}^{n_{a}} a_{i}(\phi) R_{i, j, k, 1}(\phi) \\
& \leq P_{j, k}(\phi),
\end{aligned}
$$

i.e.,

$$
P_{j, k}(\phi) \geq I
$$

Similarly, one has that $Q_{j, k}(\phi) \geq \varepsilon I$. Since $\varepsilon>0$, it follows from (20) that

$$
\operatorname{spec}\left(D_{j, k}(\phi)\right) \subset\{\lambda \in \mathbb{C}: \sigma(w, \lambda)>0\} .
$$

Hence, (19) implies that

$$
\operatorname{spec}\left(\Omega_{k}\left(A\left(g_{j}(\phi), \phi\right)\right)\right) \subset\{\lambda \in \mathbb{C}: \sigma(w, \lambda)>0\}
$$

From (15) it follows that

$$
\mu\left(A\left(g_{j}(\phi), \phi\right)\right)<w
$$

and, therefore, $\mu^{*}<w$.

Theorem 1 provides a condition for establishing whether a given scalar $w$ is an upper bound of the sought $\mu^{*}$ in Case I. This condition requires to check the existence of the positive scalar $\varepsilon$ and symmetric matrix polynomials $P_{j, k}(\phi)$ and $R_{i, j, k, l}(\phi)$ satisfying (22). For chosen degrees of such matrix polynomials, (22) is an LMI feasibility test (and, hence, a convex optimization problem [18]). Indeed, establishing whether a symmetric matrix polynomial depending affine linearly on some decision variables is SOS is equivalent to establishing feasibility of an LMI, see for instance [19]-[23] and references therein.

The LMI condition provided by Theorem 1 is based on a special case of the Positivstellensatz [24], and is sufficient for any chosen degrees of the symmetric matrix polynomials $P_{j, k}(\phi)$ and $R_{i, j, k, l}(\phi)$. The conservatism of the condition can be decreased by increasing the degrees of these matrix polynomials, see for instance [23] and references therein for results about the necessity of the Positivstellensatz.

Hereafter we will assume that the degrees of the symmetric matrix polynomials in the LMI condition provided by Theorem 1 are selected as follows. First, one arbitrarily chooses the degree 
of $P_{j, k}(\phi)$, which defines the Lyapunov function candidate. Then, the degrees of $R_{i, j, k, l}(\phi)$ are automatically selected as the largest degrees for which $P_{j, k}(\phi)-S_{j, k, 1}(\phi)-I$ and $Q_{j, k}(\phi)-$ $S_{j, k, 2}(\phi)-\varepsilon I$ have their minimum degree. Let us observe that this rule allows one to arbitrarily increase the degrees of all the symmetric matrix polynomials in (22) by increasing the degree of $P_{j, k}(\phi)$.

Hence, for a chosen degree of the symmetric matrix polynomial $P_{j, k}(\phi)$, let us define

$$
\mu_{I}=\max _{\substack{j=1, \ldots, n_{g} \\ k=1, \ldots, n}} w_{j, k}
$$

where

$$
\begin{gathered}
w_{j, k}=\inf _{w \in\left(w_{\min }, \infty\right)} w \\
\text { s.t. } \quad \exists \varepsilon>0, P_{j, k}(\phi), R_{i, j, k, l}(\phi): \text { (22) holds } \\
\quad \forall i \in\left\{1, \ldots, n_{a}\right\} \forall l \in\{1,2\} .
\end{gathered}
$$

From Theorem 1 it follows that

$$
\mu^{*} \leq \mu_{I}
$$

Indeed, $\mu_{I}$ is the best upper bound of $\mu^{*}$ provided by Theorem 1 for chosen degrees of the symmetric matrix polynomials $P_{j, k}(\phi)$ and $R_{i, j, k, l}(\phi)$. Let us observe that the quantities $w_{j, k}$ in (26) can be computed through a bisection search on $w$ where the LMI condition (22) is checked for any fixed value of $w$.

\section{B. Case II}

In this section we consider any possibility, i.e., either (10) holds for some polynomial functions $g_{j}(\phi)$ or not. The following example illustrates a situation where such polynomial functions do not exist.

Example 2. Let us consider (1) with

$$
f(x, u)=\left(\begin{array}{c}
x_{1}^{4}+x_{2}^{4}-u \\
1+x_{1}+3 x_{2}+3 x_{1} x_{2}-2 x_{2} u
\end{array}\right) .
$$

The set $\Phi$ and its expression in (8) are chosen as

$$
\left\{\begin{aligned}
\Phi & =[0,2] \\
a_{1}(\phi) & =2 \phi-\phi^{2}
\end{aligned}\right.
$$




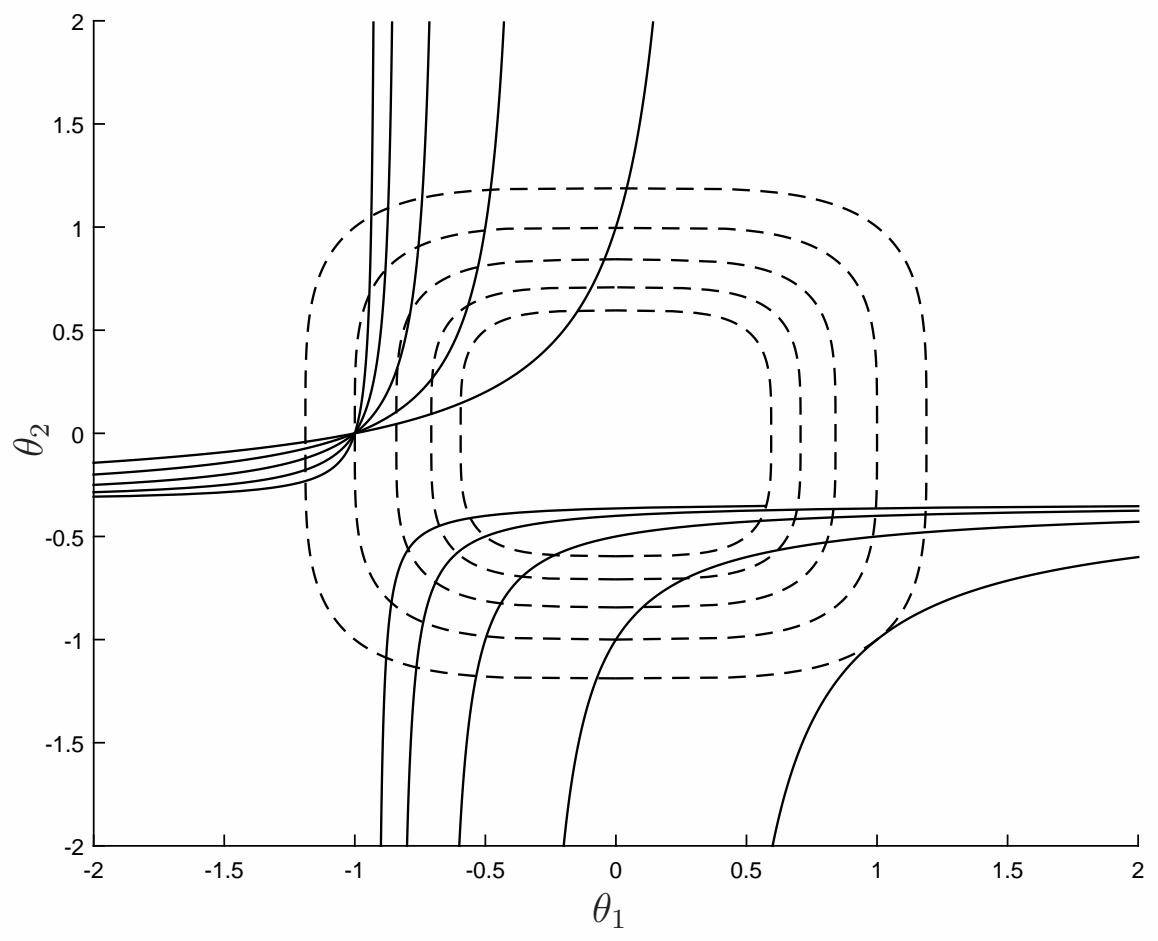

Fig. 1. Example 2. Zero level sets of $f_{1}(\theta, \phi)$ (dashed line) and $f_{2}(\theta, \phi)$ (solid line) for some values of $\phi \in \Phi$. The intersection between the two level sets are admissible equilibrium points.

It follows that the set of equilibrium points $\Theta(\phi)$ in (2) cannot be written as in (10) for polynomial functions $g_{j}(\phi)$. Figure 1 shows the zero level sets of $f(\theta, \phi)$ for $\phi \in \Phi$. Figure 2 shows the equilibrium points in $\Theta(\phi)$ for some values of $\phi \in \Phi$. Figures 3-4 show the first and second entries, respectively, of the equilibrium points in Figure 2 versus $\phi$.

In order to determine $\mu^{*}$ in (9) in this case, let us proceed as follows. For $k \in\{1, \ldots, n\}$ and $w \in \mathbb{R}$ let us define the matrix polynomials

$$
E_{k}(\theta, \phi)=\Omega_{k}(A(\theta, \phi))-w I
$$

Moreover, for symmetric matrix polynomials $T_{k}: \mathbb{R}^{n} \times \mathbb{R}^{m} \rightarrow \mathbb{R}^{c_{k} \times c_{k}}$, let us define

$$
U_{k}(\theta, \phi)=-T_{k}(\theta, \phi) E_{k}(\theta, \phi)-E_{k}(\theta, \phi)^{\prime} T_{k}(\theta, \phi) .
$$

Lastly, let us introduce the following definition: a symmetric matrix polynomial $H: \mathbb{R}^{n} \times \mathbb{R}^{m} \rightarrow$ $\mathbb{R}^{h \times h}$ is said to be sum of squares (SOS) if there exist matrix polynomials $H_{i}: \mathbb{R}^{n} \times \mathbb{R}^{m} \rightarrow \mathbb{R}^{h \times h}$, 


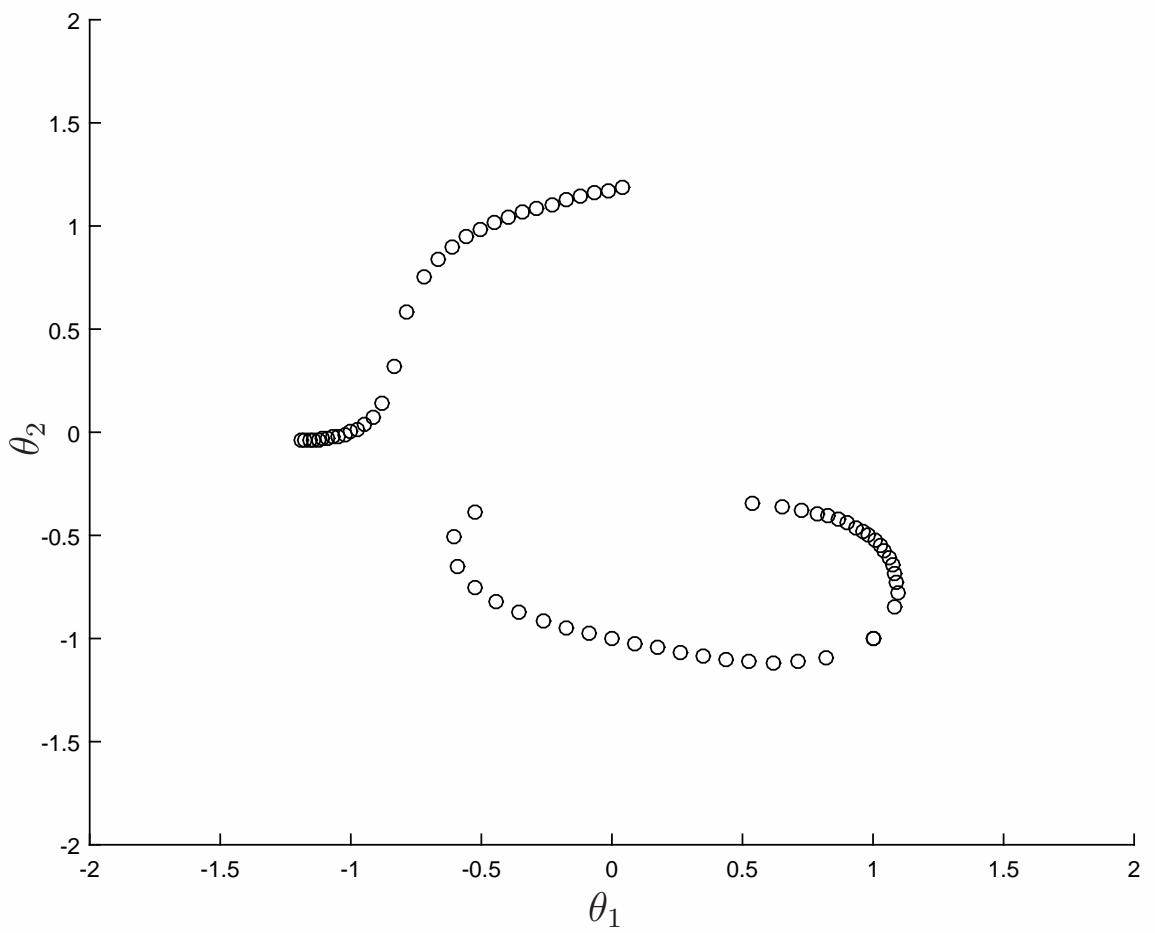

Fig. 2. Example 2. Equilibrium points in $\Theta(\phi)$ for some values of $\phi \in \Phi$.

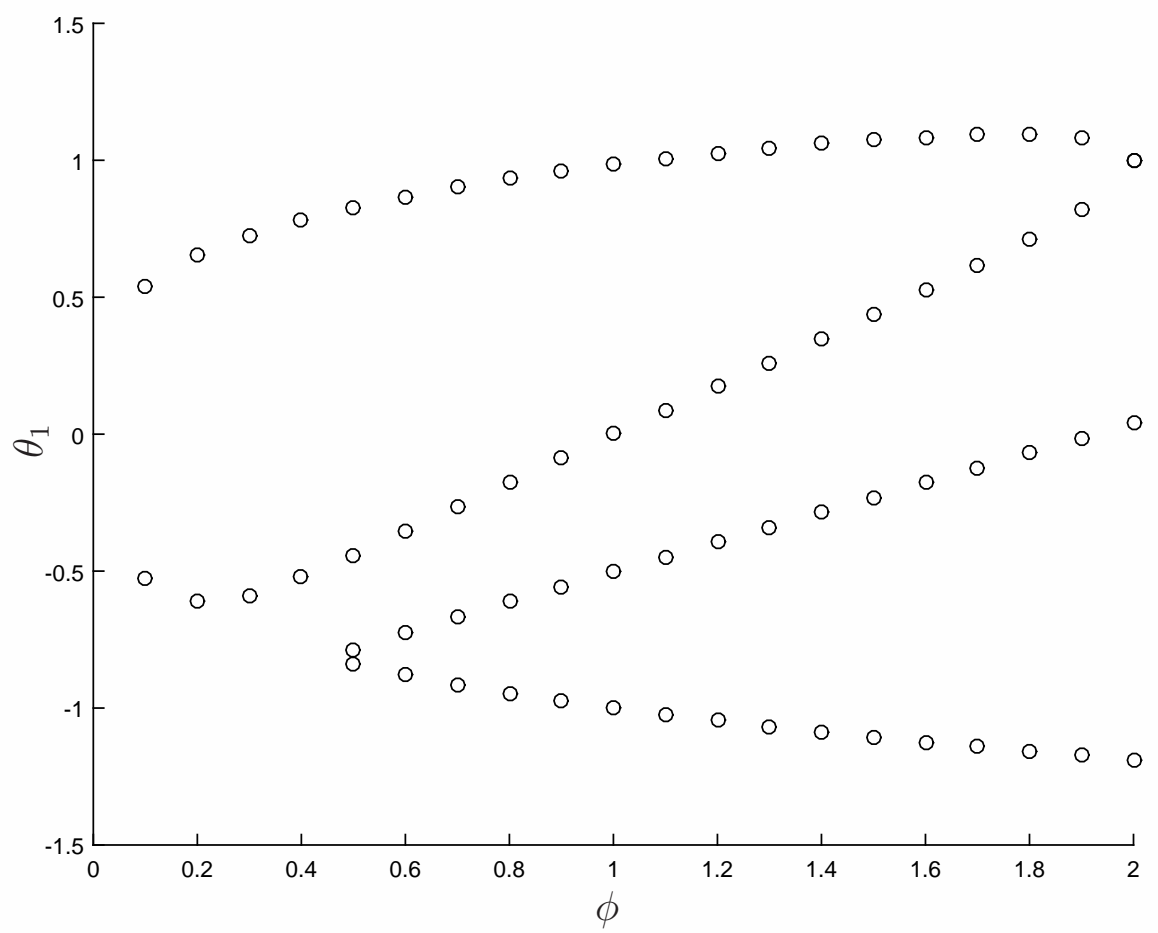

Fig. 3. Example 2. First entry of the equilibrium points in Figure 2 versus $\phi$. 


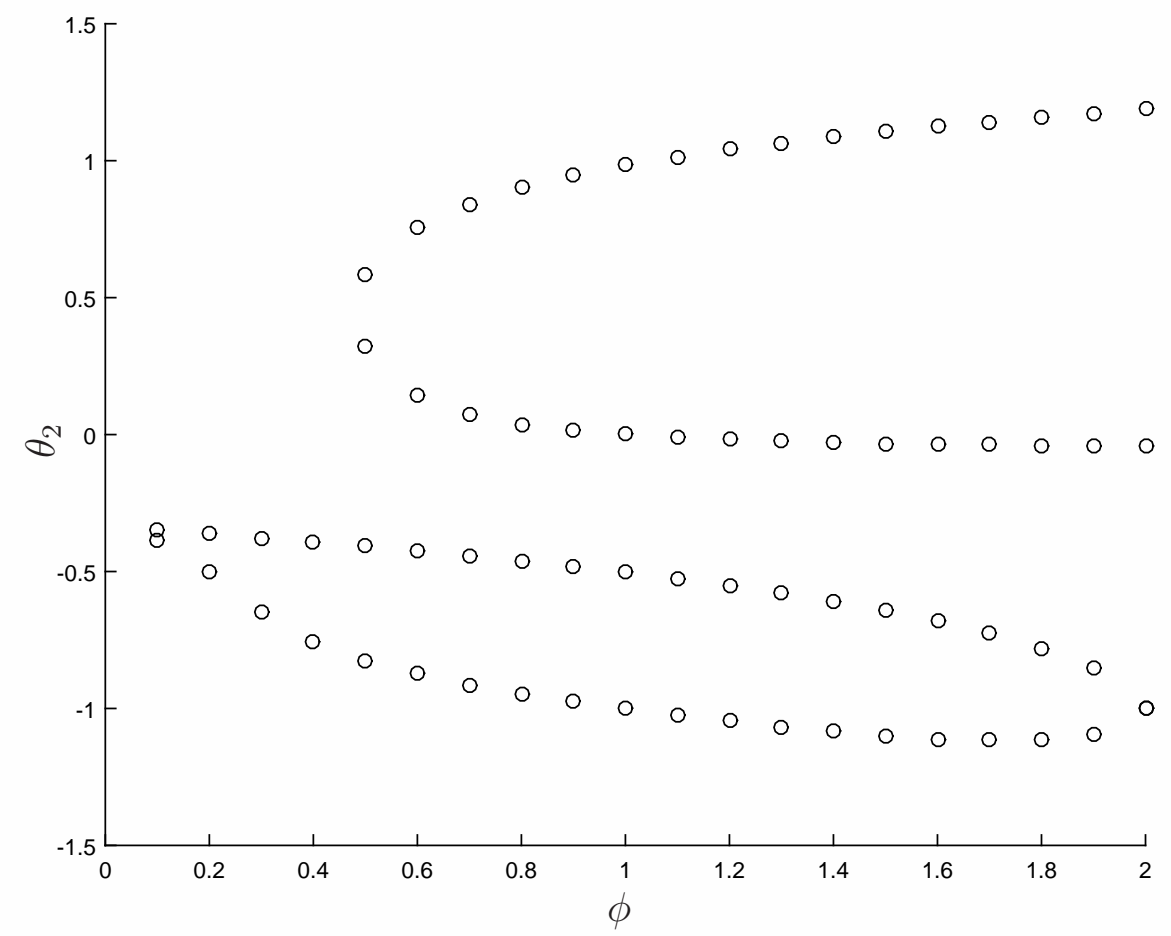

Fig. 4. Example 2. Second entry of the equilibrium points in Figure 2 versus $\phi$.

$i=1, \ldots, i_{\max }$, such that

$$
H(\theta, \phi)=\sum_{i=1}^{i_{\max }} H_{i}(\theta, \phi)^{\prime} H_{i}(\theta, \phi) .
$$

The following result provides a condition for establishing an upper bound of $\mu^{*}$ based on a convex optimization problem.

Theorem 2: Let $w \in\left(w_{\min }, \infty\right)$. Let us suppose that there exist $\varepsilon>0$ and symmetric matrix polynomials $T_{k}, V_{j, k, l}, W_{i, k, l}: \mathbb{R}^{n} \times \mathbb{R}^{m} \rightarrow \mathbb{R}^{c_{k} \times c_{k}}, i \in\left\{1, \ldots, n_{a}\right\}, j, k \in\{1, \ldots, n\}$ and $l \in\{1,2\}$, such that

$$
\left.\begin{array}{l}
T_{k}(\theta, \phi)-X_{k, 1}(\theta, \phi)-I \\
U_{k}(\theta, \phi)-X_{k, 2}(\theta, \phi)-\varepsilon I \\
W_{i, k, l}(\theta, \phi)
\end{array}\right\} \text { are SOS }
$$


for all $i \in\left\{1, \ldots, n_{a}\right\}, k \in\{1, \ldots, n\}$ and $l \in\{1,2\}$, where

$$
\begin{aligned}
X_{k, l}(\theta, \phi)= & \sum_{j=1}^{n} v_{j}(\theta, \phi)\left(f_{j}(\theta, \phi)-w_{\min } \theta_{j}\right) V_{j, k, l}(\theta, \phi) \\
& -\sum_{i=1}^{n_{a}} a_{i}(\phi) W_{i, k, l}(\theta, \phi)
\end{aligned}
$$

and $v_{j}(\theta, \phi)$ are arbitrarily chosen polynomials (their role will be investigated in Theorem 5). Then,

$$
\mu^{*}<w
$$

Proof. Suppose that (33) holds. Let $i \in\left\{1, \ldots, n_{a}\right\}, k \in\{1, \ldots, n\}, l \in\{1,2\}, \theta \in \mathbb{R}^{n}$ and $\phi \in \mathbb{R}^{m}$. From (32) it follows that

$$
\left\{\begin{array}{c}
T_{k}(\theta, \phi)-X_{k, 1}(\theta, \phi)-I \geq 0 \\
U_{k}(\theta, \phi)-X_{k, 2}(\theta, \phi)-\varepsilon I \geq 0 \\
W_{i, k, l}(\theta, \phi) .
\end{array}\right.
$$

Let $\phi \in \Phi$ and $\theta \in \Theta(\phi)$. Since $W_{k, i}(\theta, \phi) \geq 0, a_{i}(\phi) \geq 0$ and $f_{j}(\theta, \phi)-w_{\min } \theta_{j}=0$, it follows that

$$
\begin{aligned}
I \leq & T_{k}(\theta, \phi)-X_{k, 1}(\theta, \phi) \\
= & T_{k}(\theta, \phi)-\sum_{j=1}^{n} v_{j}(\theta, \phi)\left(f_{j}(\theta, \phi)-w_{\min } \theta_{j}\right) V_{j, k, l}(\theta, \phi) \\
& -\sum_{i=1}^{n_{a}} a_{i}(\phi) W_{i, k, l}(\theta, \phi) \\
\leq & T_{k}(\theta, \phi),
\end{aligned}
$$

i.e.,

$$
T_{k}(\theta, \phi) \geq I .
$$

Similarly, one has that $U_{k}(\theta, \phi) \geq \varepsilon I$. Since $\varepsilon>0$, it follows from (31) that

$$
\operatorname{spec}\left(E_{k}(\theta, \phi)\right) \subset\{\lambda \in \mathbb{C}: \sigma(w, \lambda)>0\} .
$$

Hence, (30) implies that

$$
\operatorname{spec}\left(\Omega_{k}(A(\theta, \phi))\right) \subset\{\lambda \in \mathbb{C}: \sigma(w, \lambda)>0\} .
$$

From (15) it follows that

$$
\mu(A(\theta, \phi))<w
$$


and, therefore, $\mu^{*}<w$.

Theorem 2 provides a condition for establishing whether a given scalar $w$ is an upper bound of the sought $\mu^{*}$ in Case II. This condition requires to check the existence of the positive scalar $\varepsilon>0$ and symmetric matrix polynomials $T_{k}(\theta, \phi), V_{j, k, l}(\theta, \phi)$ and $W_{i, k, l}(\theta, \phi)$ satisfying (33). For chosen degrees of such matrix polynomials, (33) is an LMI feasibility test analogously to (22) in Theorem 1.

The condition provided by Theorem 2 is sufficient for any chosen degrees of the symmetric matrix polynomials $T_{k}(\theta, \phi), V_{j, k, l}(\theta, \phi)$ and $W_{i, k, l}(\theta, \phi)$. The conservatism of the condition can be decreased by increasing the degrees of these matrix polynomials as discussed for Theorem 1.

Hereafter we will assume that the degrees of the symmetric matrix polynomials in the LMI condition provided by Theorem 2 are selected as follows. First, one arbitrarily chooses the degree of $T_{k}(\theta, \phi)$, which defines the Lyapunov function candidate. Then, the degrees of $V_{j, k, l}(\theta, \phi)$ and $W_{i, k, l}(\theta, \phi)$ are automatically selected as the largest degrees for which $T_{k}(\theta, \phi)-X_{k, 1}(\theta, \phi)-I$ and $U_{k}(\theta, \phi)-X_{k, 2}(\theta, \phi)-\varepsilon I$ have their minimum degree. Let us observe that this rule allows one to arbitrarily increase the degrees of all the symmetric matrix polynomials in (33) by increasing the one of $T_{k}(\theta, \phi)$.

Hence, for a chosen degree of the symmetric matrix polynomial $T_{k}(\theta, \phi)$, let us define

$$
\mu_{I I}=\max _{k=1, \ldots, n} w_{k}
$$

where

$$
\begin{aligned}
w_{k}= & \inf _{w \in\left(w_{\text {min }}, \infty\right)} w \\
\text { s.t. } & \exists \varepsilon>0, T_{k}(\theta, \phi), V_{j, k, l}(\theta, \phi), W_{i, k, l}(\theta, \phi): \\
& \text { (33) holds } \forall i \in\left\{1, \ldots, n_{a}\right\} \forall l \in\{1,2\} .
\end{aligned}
$$

From Theorem 2 it follows that

$$
\mu^{*} \leq \mu_{I I}
$$

Indeed, $\mu_{I I}$ is the best upper bound of $\mu^{*}$ provided by Theorem 2 for chosen degrees of the symmetric matrix polynomials $T_{k}(\theta, \phi), V_{j, k, l}(\theta, \phi)$ and $W_{i, k, l}(\theta, \phi)$. Let us observe that the quantities $w_{k}$ in (37) can be computed through a bisection search on $w$ where the LMI condition (33) is checked for any fixed value of $w$. 


\section{Conservatism And Discrete-Time}

In this section we investigate the conservatism of the upper bounds of $\mu^{*}$ derived in the previous section. Moreover, we show how the proposed methodology can be extended to the case of discrete-time nonlinear systems.

\section{A. Conservatism: Case I}

The following result states that, under some mild assumptions on $\Phi$, the LMI condition provided by Theorem 1 is not only sufficient but also necessary and, consequently, the upper bound $\mu_{I}$ is asymptotically nonconservative.

Theorem 3: Let us suppose that $\Phi$ is compact and that the polynomials $a_{i}(\phi)$ have even degree and that their highest degree forms have no common zeros except 0 . Then, $\mu^{*}<w$ with $w \in\left(w_{\min }, \infty\right)$ if and only if there exist $\varepsilon>0$ and symmetric matrix polynomials $P_{j, k}(\phi)$ and $R_{i, j, k, l}(\phi)$ such that (22) holds. Consequently, for all $\tilde{\varepsilon}>0$ there exist a sufficiently large degree of $P_{j, k}(\phi)$ such that

$$
0 \leq \mu_{I}-\mu^{*} \leq \tilde{\varepsilon}
$$

Proof. The sufficiency has been proven in Theorem 1, let us hence consider the necessity. Suppose that $\mu^{*}<w$ with $w \in\left(w_{\min }, \infty\right)$. Let $\phi \in \Phi$. It follows that

$$
\mu\left(A\left(g_{j}(\phi), \phi\right)\right)<w \quad \forall j \in\left\{1, \ldots, n_{g}\right\}
$$

Let $j \in\left\{1, \ldots, n_{g}\right\}$ and $k \in\{1, \ldots, n\}$. From (15) one has that

$$
\operatorname{spec}\left(\Omega_{k}\left(A\left(g_{j}(\phi), \phi\right)\right)\right) \subset\{\lambda \in \mathbb{C}: \sigma(w, \lambda)>0\} .
$$

This implies that

$$
\operatorname{spec}\left(D_{j, k}(\phi)\right) \subset\left\{\lambda \in \mathbb{C}: \sigma\left(w_{\text {min }}, \lambda\right)>0\right\}
$$

Hence, the Lyapunov equation

$$
Q_{j, k}(\phi)=I
$$

admits a unique solution $P_{j, k}(\phi)$ that satisfies

$$
P_{j, k}(\phi) \geq \varepsilon_{1} I
$$


for some $\varepsilon_{1}>0$. Since $Q_{j, k}(\phi)$ is a polynomial function, it follows that $P_{j, k}(\phi)$ is a rational function that can be expressed as

$$
P_{j, k}(\phi)=\frac{P_{j, k, \text { num }}(\phi)}{P_{j, k, \text { den }}(\phi)}
$$

where

$$
P_{j, k, d e n}(\phi)>\varepsilon_{2}
$$

for some $\varepsilon_{2}>0$. Let us redefine $P_{j, k}(\phi)$ as

$$
P_{j, k}(\phi) \leftarrow \varepsilon_{3} P_{j, k, \text { num }}(\phi)
$$

where $\varepsilon_{3}=\max \left\{1, \varepsilon_{1}^{-1}\right\}$. It follows that $P_{j, k}(\phi)$ is a symmetric matrix polynomial that satisfies

$$
\left\{\begin{aligned}
P_{j, k}(\phi)-I & \geq 0 \\
Q_{j, k}(\phi)-\varepsilon_{2} \varepsilon_{3} I & \geq 0 .
\end{aligned}\right.
$$

Since $\Phi$ is compact, and since the polynomials $a_{i}(\phi)$ have even degree and that their highest degree forms have no common zeros except 0, it follows from Putinar's Positivstellensatz [25] that there exist symmetric matrix polynomials $R_{i, j, k, l}(\phi)$ such that (22) holds for some $\varepsilon>0$.

The next result provides a sufficient and necessary condition for establishing whether the upper bound $\mu_{I}$ is tight.

Theorem 4: Without loss of generality, suppose that $\mu_{I}>0$. Then,

$$
\mu^{*}=\mu_{I}
$$

if there exist $j \in\left\{1, \ldots, n_{g}\right\}, k \in\{1, \ldots, n\}$ and $\phi \in \mathcal{Z}_{j, k}$ such that

$$
\left\{\begin{aligned}
\mu\left(A\left(g_{j}(\phi), \phi\right)\right. & =\mu_{I} \\
\phi & \in \Phi
\end{aligned}\right.
$$

where

$$
\mathcal{Z}_{j, k}=\left\{\phi \in \mathbb{R}^{m}: \lambda_{\min }\left(\hat{Q}_{j, k}(\phi)-\hat{S}_{j, k, 2}(\phi)\right)=0\right\}
$$

and $\hat{Q}_{j, k}(\phi)$ and $\hat{S}_{j, k, 2}(\phi)$ are the matrices $Q_{j, k}(\phi)$ and $S_{j, k, 2}(\phi)$ evaluated for the optimal values of $w, P_{j, k}(\phi)$ and $R_{i, j, k, l}(\phi)$ in (26). Moreover, if $\Phi$ is compact, this condition is not only sufficient but also necessary. 
Proof. " $\Leftarrow$ " Suppose that $(41)$ holds. From the definition of $\mu_{I}$ this implies that

$$
\mu_{I} \leq \mu^{*}
$$

Hence, from (27) it follows that $\mu^{*}=\mu_{I}$.

“ $\Rightarrow$ " Suppose that $\Phi$ is compact and $\mu^{*}=\mu_{I}$. From the continuity of $\mu\left(A\left(g_{j}(\phi), \phi\right)\right.$ it follows that there exists $\phi^{*} \in \Phi$ such that (41) holds for some $j \in\left\{1, \ldots, n_{g}\right\}$ with $\phi=\phi^{*}$. Let us consider hereafter such a value of $j$, and let $k \in\{1, \ldots, n\}$ be such that

$$
\mu^{*}=\sum_{i=1}^{k} \lambda_{z_{i}}\left(A\left(g_{j}\left(\phi^{*}\right), \phi^{*}\right)\right.
$$

Let us observe that

$$
\exists i \in\left\{1, \ldots, c_{k}\right\}: \lambda_{i}\left(\Omega_{k}\left(A\left(g_{j}\left(\phi^{*}\right), \phi^{*}\right)\right)\right)=\mu^{*} .
$$

Since $w_{j, k}=\mu^{*}$ for the considered values of $j$ and $k$, one has

$$
\exists i \in\left\{1, \ldots, c_{k}\right\}: \Re\left(\lambda_{i}\left(D_{j, k}\left(\phi^{*}\right)\right)\right)=0 .
$$

Since $P_{j, k}(\phi)-S_{j, k, 1}(\phi)-I$ and $R_{i, j, k, 1}(\phi)$ are SOS, and since $a_{i}\left(\phi^{*}\right) \geq 0$, it follows that

$$
\hat{P}_{j, k}\left(\phi^{*}\right) \geq I
$$

where $\hat{P}_{j, k}(\phi)$ is the optimal value of $P_{j, k}(\phi)$ in (26). Since $\hat{Q}_{j, k}(\phi)-\hat{S}_{j, k, 2}(\phi)$ is SOS, this implies that

$$
\lambda_{\min }\left(\hat{Q}_{j, k}\left(\phi^{*}\right)-\hat{S}_{j, k, 2}\left(\phi^{*}\right)\right)=0,
$$

i.e., $\phi^{*} \in \mathcal{Z}_{j, k}$.

Theorem 4 provides a sufficient condition for establishing whether the computed upper bound $\mu_{I}$ is tight, which is also necessary under the mild assumption that $\Phi$ is compact. The condition provided by Theorem 4 consists of checking whether (41) holds for some $j \in\left\{1, \ldots, n_{g}\right\}$, $k \in\{1, \ldots, n\}$ and $\phi \in \mathcal{Z}_{j, k}$. For any admissible values of $j$ and $k$, this can be done by computing $\mathcal{Z}_{j, k}$ (e.g., through linear algebra operations, see for instance [14] and references therein), and then by trivial substitution of $\phi$ into (41). 


\section{B. Conservatism: Case II}

The following result states that, under some mild assumptions on $\Phi$ and some assumptions on $\Theta(\phi)$, the LMI condition provided by Theorem 2 is not only sufficient but also necessary and, consequently, the upper bound $\mu_{I I}$ is asymptotically nonconservative.

Theorem 5: Let us suppose that $\Phi$ is compact and $\Theta(\phi)$ is compact for all $\phi \in \Phi$. Let us also suppose that the polynomials $a_{i}(\phi)$ and $v_{j}(\theta, \phi)\left(f_{j}(\theta, \phi)-w_{\min } \theta_{j}\right)$ have even degree and that their highest degree forms have no common zeros except 0 , where $v_{j}(\theta, \phi)$ can be arbitrarily chosen under the constraint that it is positive over $\Theta(\phi) \times \Phi$ for all $\phi \in \Phi$. Then, $\mu^{*}<w$ with $w \in\left(w_{\min }, \infty\right)$ if and only if there exist $\varepsilon>0$ and symmetric matrix polynomials $T_{k}(\theta, \phi), V_{j, k, l}(\theta, \phi)$ and $W_{i, k, l}(\theta, \phi)$ such that (33) holds. Consequently, for all $\tilde{\varepsilon}>0$ there exist a sufficiently large degree of $T_{k}(\theta, \phi)$ such that

$$
0 \leq \mu_{I I}-\mu^{*} \leq \tilde{\varepsilon}
$$

Proof. The sufficiency has been proven in Theorem 2, let us hence consider the necessity. Suppose that $\mu^{*}<w$ with $w \in\left(w_{\min }, \infty\right)$. Let $\phi \in \Phi$ and $\theta \in \Theta(\phi)$. It follows that

$$
\mu(A(\theta, \phi))<w
$$

Let $k \in\{1, \ldots, n\}$. Proceeding as in the proof of Theorem 1, it follows that there exist symmetric matrix polynomials $T_{k}(\theta, \phi)$ that satisfy

$$
\left\{\begin{aligned}
T_{k}(\theta, \phi)-I & \geq 0 \\
U_{k}(\theta, \phi)-\varepsilon_{2} \varepsilon_{3} I & \geq 0
\end{aligned}\right.
$$

for some $\varepsilon_{2}>0$ and $\varepsilon_{3}>0$. Since $\Phi$ is compact and $\Theta(\phi)$ is compact for all $\phi \in \Phi$, and since $a_{i}(\phi)$ and $v_{j}(\theta, \phi)\left(f_{j}(\theta, \phi)-w_{\min } \theta_{j}\right)$ have even degree and that their highest degree forms have no common zeros except 0, it follows from Putinar's Positivstellensatz [25] that there exist symmetric matrix polynomials $V_{j, k, l}(\theta, \phi)$ and $W_{i, k, l}(\theta, \phi)$ such that (33) holds for some $\varepsilon>0$.

The following result provides a sufficient and necessary condition for establishing whether the upper bound $\mu_{I I}$ is tight. 
Theorem 6: Without loss of generality, suppose that $\mu_{I I}>0$. Then,

$$
\mu^{*}=\mu_{I I}
$$

if there exist $k \in\{1, \ldots, n\}$ and $(\theta, \phi) \in \mathcal{Z}_{k}$ such that

$$
\left\{\begin{aligned}
\mu(A(\theta, \phi) & =\mu_{I I} \\
f(\theta, \phi) & =0 \\
\phi & \in \Phi
\end{aligned}\right.
$$

where

$$
\begin{aligned}
\mathcal{Z}_{k}= & \left\{(\theta, \phi) \in \mathbb{R}^{n} \times \mathbb{R}^{m}: \lambda_{\min }\left(\hat{U}_{k}(\theta, \phi)\right.\right. \\
& \left.\left.-\hat{X}_{k, 2}(\theta, \phi)\right)=0\right\}
\end{aligned}
$$

and $\hat{U}_{k}(\theta, \phi)$ and $\hat{X}_{k, 2}(\theta, \phi)$ are the matrices $U_{k}(\theta, \phi)$ and $X_{k, 2}(\theta, \phi)$ evaluated for the optimal values of $w, T_{k}(\theta, \phi), V_{j, k, l}(\theta, \phi)$ and $W_{i, k, l}(\theta, \phi)$ in (37). Moreover, if $\Phi$ is compact and $\Theta(\phi)$ is compact for all $\phi \in \Phi$, this condition is not only sufficient but also necessary.

Proof. " $\Leftarrow$ " Suppose that (45) holds. From the definition of $\mu_{I I}$ this implies that

$$
\mu_{I I} \leq \mu^{*}
$$

Hence, from (38) it follows that $\mu^{*}=\mu_{I I}$.

“ $\Rightarrow$ " Suppose that $\Phi$ is compact, $\Theta(\phi)$ is compact for all $\phi \in \Phi$, and $\mu^{*}=\mu_{I I}$. From the continuity of $\mu\left(A(\theta, \phi)\right.$ it follows that there exist $\phi^{*} \in \Phi$ and $\theta \in \Theta\left(\phi^{*}\right)$ such that (45) holds with $\theta=\theta^{*}$ and $\phi=\phi^{*}$. Let $k \in\{1, \ldots, n\}$ be such that

$$
\mu^{*}=\sum_{i=1}^{k} \lambda_{z_{i}}\left(A\left(\theta^{*}, \phi^{*}\right) .\right.
$$

Let us observe that

$$
\exists i \in\left\{1, \ldots, c_{k}\right\}: \lambda_{i}\left(\Omega_{k}\left(A\left(\theta^{*}, \phi^{*}\right)\right)\right)=\mu^{*}
$$

Since $w_{k}=\mu^{*}$ for the considered value of $k$, one has

$$
\exists i \in\left\{1, \ldots, c_{k}\right\}: \Re\left(\lambda_{i}\left(E_{k}\left(\theta^{*}, \phi^{*}\right)\right)\right)=0 .
$$

Since $T_{k}(\theta, \phi)-X_{k, 1}(\theta, \phi)-I$ and $W_{i, k, l}(\phi)$ are SOS, and since $a_{i}\left(\phi^{*}\right) \geq 0$ and $f_{j}\left(\theta^{*}, \phi^{*}\right)-$ $w_{\min } \theta_{j}^{*}=0$, it follows that

$$
\hat{T}_{k}\left(\theta^{*}, \phi^{*}\right) \geq I
$$


where $\hat{T}_{k}(\theta, \phi)$ is the optimal value of $T_{k}(\theta, \phi)$ in (37). Since $\hat{U}_{k}(\theta, \phi)-\hat{X}_{k, 2}(\theta, \phi)$ is SOS, this implies that

$$
\lambda_{\min }\left(\hat{U}_{k}\left(\theta^{*}, \phi^{*}\right)-\hat{X}_{k, 2}\left(\theta^{*}, \phi^{*}\right)\right)=0,
$$

i.e., $\left(\theta^{*}, \phi^{*}\right) \in \mathcal{Z}_{k}$.

Theorem 6 provides a sufficient condition for establishing whether the computed upper bound $\mu_{I I}$ is tight, which is also necessary under the assumptions that $\Phi$ is compact and $\Theta(\phi)$ is compact for all $\phi \in \Phi$. The condition provided by Theorem 6 consists of checking whether (45) holds for some $k \in\{1, \ldots, n\}$ and $(\theta, \phi) \in \mathcal{Z}_{k}$. This can be done through linear algebra operations as explained for the condition of Theorem 4.

\section{Discrete-Time}

In this subsection we show how the proposed methodology can be extended to the case of discrete-time nonlinear systems. Specifically, we consider

$$
x(t+1)=f(x(t), u(t))
$$

where $t \in \mathbb{R}, x \in \mathbb{R}^{n}, u \in \mathbb{R}^{m}$, and $f(x(t), u(t))$ is a polynomial function in $x(t)$ and $u(t)$. For brevity, we only report the expressions of the previous sections that need to be changed.

Let us start by considering the problem formulation in Section II. The set of equilibrium points $\Theta(\phi)$ in (2) is replaced by

$$
\Theta(\phi)=\left\{\theta \in \mathbb{R}^{n}: f(\theta, \phi)-\theta=0\right\} .
$$

The instability measure $\mu(M)$ in (6) is replaced by

$$
\mu(M)=\prod_{i=1}^{n} \max \left\{1,\left|\lambda_{i}(M)\right|\right\} .
$$

Hence, let us consider the upper bounds in Section III. The condition (15) that defines the matrix function $\Omega_{k}(M)$ is replaced by

$$
\operatorname{spec}\left(\Omega_{k}(M)\right)=\left\{\prod_{i=1}^{k} \lambda_{z_{i}}(M), z \in \mathcal{I}_{k}\right\} .
$$


The quantities (17)-(20) introduced for Case I are replaced by

$$
\left\{\begin{aligned}
w_{\min } & =1 \\
\sigma(w, \lambda) & =1-\frac{|\lambda|}{w} \\
D_{j, k}(\phi) & =\frac{\Omega_{k}\left(A\left(g_{j}(\phi), \phi\right)\right)}{w} \\
Q_{j, k}(\phi) & =P_{j, k}(\phi)-D_{j, k}(\phi)^{\prime} P_{j, k}(\phi) D_{j, k}(\phi) .
\end{aligned}\right.
$$

The quantities (30)-(31) introduced for Case II are replaced by

$$
\left\{\begin{aligned}
E_{k}(\theta, \phi) & =\frac{\left.\Omega_{k}(\theta, \phi)\right)}{w} \\
U_{k}(\theta, \phi) & =T_{k}(\theta, \phi)-E_{k}(\theta, \phi)^{\prime} T_{k}(\theta, \phi) E_{k}(\theta, \phi) .
\end{aligned}\right.
$$

The following corollary states that the theorems derived in the previous sections for the case continuous-time nonlinear system (1) can be readily used for the discrete-time nonlinear system (47).

Corollary 1: Theorems 1-6 hold for the discrete-time nonlinear system in (47) instead of the continuous-time nonlinear system in (1) by performing the changes (48)-(52).

Proof. Analogous to the proofs of Theorems 1-6 by replacing the stability conditions based on eigenvalues and Lyapunov functions.

We conclude this section reporting the expression of $\Omega_{k}(M)$ in a few cases. In particular, for $n=3$ one has

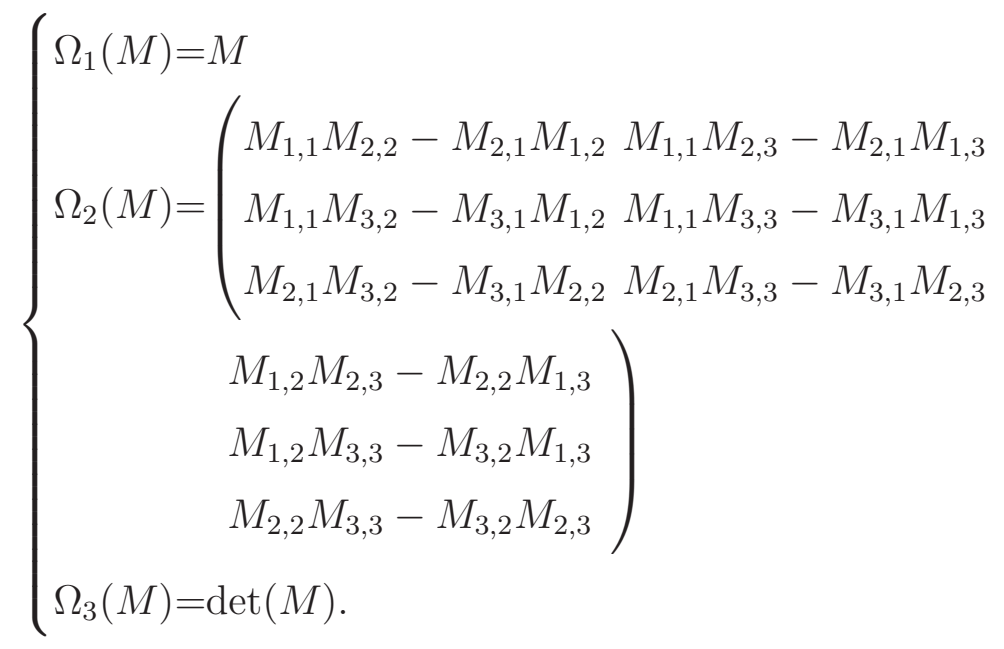




\section{EXAMPLES}

In this section we present some illustrative examples of the proposed results. The computations have been done on a standard personal computer (Windows 8, Intel Core i7, $3.4 \mathrm{GHz}, 8 \mathrm{~GB}$ RAM) with Matlab using the toolbox SeDuMi [26]. The degree of $R_{i, j, k, l}(\phi)$ in the LMI (22) is automatically chosen from the one of $P_{j, k}(\phi)$ as described after Theorem 1. Similarly, the degrees of $V_{j, k, l}(\theta, \phi)$ and $W_{i, k, l}(\theta, \phi)$ in the LMI (33) are automatically chosen from the one of $T_{k}(\theta, \phi)$ as described after Theorem 2.

\section{A. Example 1 (Continued)}

Let us consider consider again (1) with (11)-(13). The matrix $A(\theta, \phi)$ of the linearized system (3) is

$$
A(\theta, \phi)=\left(\begin{array}{ccc}
-2 \phi_{2}^{2} & -2 \theta_{2} & -2 \\
-2 \theta_{1}+0.5 \phi_{2} & 1 & 0 \\
1+\phi_{1} & 1 & 0
\end{array}\right) .
$$

Since the set of equilibrium points $\Theta(\phi)$ in (2) can be written as in (10) for polynomial functions $g_{j}(\phi), j=1,2$, given by (13), this example can be investigated under Case I or Case II.

Let us start with Case I, and let us determine the upper bound $\mu_{I}$ in (25). This requires to determine the quantities $w_{j, k}$ in (26) for $j \in\{1,2\}$ and $k \in\{1,2,3\}$.

Let us consider first $j=1$. We have

$$
A\left(g_{1}(\phi), \phi\right)=\left(\begin{array}{ccc}
-2 \phi_{2}^{2} & 0 & -2 \\
0.5 \phi_{2} & 1 & 0 \\
1+\phi_{1} & 1 & 0
\end{array}\right) .
$$

By searching for a symmetric matrix polynomial $P_{j, k}(\phi)$ of degree 1 , we obtain

$$
w_{1,1}=1.252, \quad w_{2,1}=1.361, \quad w_{3,1}=1.000 .
$$

Then, we consider $j=2$. We have

$$
\begin{aligned}
& A\left(g_{2}(\phi), \phi\right)= \\
& \left(\begin{array}{ccc}
-2 \phi_{2}^{2} & -2-4 \phi_{1}+\phi_{2}+\phi_{1} \phi_{2}-2 \phi_{1}^{2} & -2 \\
2+2 \phi_{1}-0.5 \phi_{2} & 1 & 0 \\
1+\phi_{1} & 1 & 0
\end{array}\right) .
\end{aligned}
$$


By proceeding as in the previous case, we obtain

$$
w_{1,2}=1.252, \quad w_{2,2}=1.476, \quad w_{3,2}=1.000 .
$$

Hence, $\mu_{I}=1.476$. The computational time for establishing feasibility of the LMI condition (22) is less than 1 second.

Next, let us establish whether $\mu_{I}$ is tight. We find that (41) holds with

$$
\left\{\begin{array}{l}
j=2 \\
k=2 \\
\phi=(-0.609,-0.094)^{\prime} .
\end{array}\right.
$$

Therefore, we conclude that the upper bound is tight, i.e., $\mu^{*}=\mu_{I}$. Indeed, for $\phi=(-0.609,-0.094)^{\prime}$, one has that

$$
\mu\left(A\left(g_{2}(\phi), \phi\right)\right)=\mu_{I}
$$

that is, the upper bound $\mu_{I}$ is achieved by $\mu(A(\theta, \phi))$ for an admissible value of $\phi$ (i.e., $\left.(-0.609,-0.094)^{\prime}\right)$ and $\theta$ (i.e., $\left.g_{2}\left((-0.609,-0.094)^{\prime}\right)\right)$.

Next, let us investigate this example under Case II. It turns out that $\mu^{*}$ can be obtained through the upper bound $\mu_{I I}$. However, it is interesting that one must search for a symmetric matrix polynomial $T_{k}(\theta, \phi)$ of degree 3 , while we simply obtain $\mu_{I}=\mu^{*}$ by searching for a symmetric matrix polynomial $P_{j, k}(\phi)$ of degree 1 : the computational burden required by Case I is significantly smaller than that required by Case II.

Lastly, we compare the proposed method with a grid technique. Specifically, we generate $n_{\text {grid }}$ number of samples of $\phi$ equally distributed in $\Phi=[-1,1]^{2}$. Then, for each sample of $\phi$, we evaluate the instability measure $\mu\left(A\left(g_{j}(\phi), \phi\right)\right)$. In order to obtain the maximizer of $\phi$ with precision at the third digit, we choose $n_{\text {grid }}=2001^{2}$, and the computational time is 346 seconds (much larger than that of the proposed method). Moreover, this provides only a lower bound of $\mu^{*}$ (while the proposed method provides guaranteed upper bounds of $\mu^{*}$ and, in this example, the exact value of $\mu^{*}$ ). 


\section{B. Example 2 (Continued)}

Let us consider again (1) with (28)-(29). The matrix $A(\theta, \phi)$ of the linearized system (3) is

$$
A(\theta, \phi)=\left(\begin{array}{cc}
2 \theta_{1} & 2 \theta_{2} \\
1+3 \theta_{2} & 2+3 \theta_{1}
\end{array}\right) .
$$

Since the set of equilibrium points $\Theta(\phi)$ in (2) cannot be written as in (10) for polynomial functions $g_{j}(\phi)$, this example can be investigated under Case II only.

Let us determine the upper bound $\mu_{I I}$ in (36). This requires to determine the quantities $w_{k}$ in (37) for $k \in\{1,2\}$. By searching for a symmetric matrix polynomial $T_{k}(\theta, \phi)$ of degree 0 , we obtain

$$
w_{1}=6.147, \quad w_{2}=8.170 .
$$

Hence, $\mu_{I I}=8.170$. The computational time for establishing feasibility of the LMI condition (33) is less than 1 second.

Next, let us establish whether $\mu_{I I}$ is tight. We find that (45) holds with

$$
\left\{\begin{aligned}
k & =2 \\
\theta & =(1.076,-0.651)^{\prime} \\
\phi & =1.518 .
\end{aligned}\right.
$$

Therefore, we conclude that the upper bound is tight, i.e., $\mu^{*}=\mu_{I I}$. Indeed, for $\theta=\left((1.076,-0.651)^{\prime}\right.$ and $\phi=1.518$, one has that

$$
\mu(A(\theta, \phi))=\mu_{I I}
$$

that is, the upper bound $\mu_{I I}$ is achieved by $\mu(A(\theta, \phi))$ for an admissible value of $\phi$ (i.e., 1.518) and $\theta$ (i.e., $\left.(1.076,-0.651)^{\prime}\right)$.

Lastly, we compare the proposed method with a grid technique. Specifically, we generate $n_{\text {grid }}$ number of samples of $\phi$ equally distributed in $\Phi=[0,2]$. Then, for each sample of $\phi$, we attempt to compute the set of equilibrium points $\Theta(\phi)$ in (2) by using the function "solve" of Matlab, and we evaluate the instability measure $\mu(A(\theta, \phi))$ for all $\theta \in \Theta(\phi)$. Some comments are as follows. 
1) The computation of the set $\Theta(\phi)$ is wrong for some values of $\phi$. For instance, with $\phi=1$, we get only the solutions $(-1,0)^{\prime}$ and $(0,-1)^{\prime}$, however other two solutions exist as it can be easily verified by graphical investigation. Indeed, as explained in Section I, no reliable method does exist for solving a system of polynomial equations.

2) In order to obtain the maximizer of $\phi$ with precision at the third digit, we choose $n_{\text {grid }}=$ 2001, and the computational time is 850 seconds (much larger than that of the proposed method). Moreover, this provides only a lower bound of $\mu^{*}$ (while the proposed method provides guaranteed upper bounds of $\mu^{*}$ and, in this example, the exact value of $\mu^{*}$ ).

\section{Example 3}

Let us consider (1) with

$$
f(x, u)=\left(\begin{array}{c}
x_{2}^{4}+x_{3}^{3}-u_{1} x_{2} \\
-2 x_{1}^{2}+x_{3}^{4}-u_{2} x_{3} \\
-4+x_{2} x_{3}+x_{1}^{4}+u_{1}+u_{2}
\end{array}\right) .
$$

The set $\Phi$ in (8) is defined by

$$
\left\{\begin{aligned}
\Phi & =[-3,3]^{2} \\
a_{i}(\phi) & =9-\phi_{i}^{2} \quad \forall i=1,2 .
\end{aligned}\right.
$$

Let us determine the upper bound $\mu_{I I}$ in (36). This requires to determine the quantities $w_{k}$ in (37) for $k \in\{1,2,3\}$. By searching for a symmetric matrix polynomial $T_{k}(\theta, \phi)$ of degree 1 , we obtain

$$
w_{1}=24.687, \quad w_{2}=24.211, \quad w_{3}=1.842 .
$$

Hence, $\mu_{I I}=24.687$. The computational time for establishing feasibility of the LMI condition (33) is 23 seconds.

Next, let us establish whether $\mu_{I I}$ is tight. We find that (45) holds with

$$
\left\{\begin{array}{l}
k=2 \\
\theta=(1.608,-1.837,-1.804)^{\prime} \\
\phi=(-3,-3)^{\prime} .
\end{array}\right.
$$


Therefore, we conclude that the upper bound is tight, i.e., $\mu^{*}=\mu_{I I}$. Indeed, for $\theta=(1.608,-1.837$, $-1.804)^{\prime}$ and $\phi=(-3,-3)^{\prime}$, one has that

$$
\mu(A(\theta, \phi))=\mu_{I I}
$$

Lastly, we compare the proposed method with a grid technique. Specifically, we generate $n_{\text {grid }}$ number of samples of $\phi$ equally distributed in $\Phi=[-3,3]^{2}$. Then, for each sample of $\phi$, we attempt to compute the set of equilibrium points $\Theta(\phi)$ in (2), and we evaluate the instability measure $\mu(A(\theta, \phi))$ for all $\theta \in \Theta(\phi)$. Some comments are as follows.

1) The computation of the set $\Theta(\phi)$ is wrong for some values of $\phi$. For instance, with $\phi=$ $(-3,-3)^{\prime}$, we get only the wrong solution $(216.543,-3.133 e+31,16.794)^{\prime}$, however at least a solution does exist, which is the maximizer found above with the proposed method. Indeed, as explained in Section I, no reliable method does exist for solving a system of polynomial equations.

2) In order to obtain the maximizer of $\phi$ with precision at the third digit, we choose $n_{\text {grid }}=$ $6001^{2}$, and the estimated ${ }^{1}$ computational time is greater than 100 days (much larger than that of the proposed method). Moreover, this provides only a lower bound of $\mu^{*}$ (while the proposed method provides guaranteed upper bounds of $\mu^{*}$ and, in this example, the exact value of $\left.\mu^{*}\right)$.

\section{CONCLUSION}

This paper has addressed the problem of determining the largest instability measure in linearized nonlinear systems, either continuous-time or discrete-time, over all admissible equilibrium points and all admissible constant inputs. It has been shown that upper bounds of the sought instability measure can be established through LMIs by searching for polynomiallydependent Lyapunov function candidates. Moreover, it has been shown that these upper bounds are nonconservative for a sufficiently large degree of the Lyapunov function candidates under some conditions. Lastly, necessary and sufficient conditions have been provided for establishing whether the obtained upper bounds are nonconservative. Two cases have been considered in the derivation of these results: first, when the equilibrium points are known polynomial functions of

\footnotetext{
${ }^{1}$ This estimate has been calculated using $n_{\text {grid }}=61^{2}$ and then scaling the obtained computational time.
} 
the input, and, second, when the equilibrium points are unknown (polynomial or non-polynomial) functions of the input.

Some numerical examples have also shown the advantages of the proposed method with respect to a grid technique in terms of guarantees and computational time. In this respect, it is fair to mention that grid techniques can also handle large scale systems while the proposed method cannot. Hence, future work could investigate the simplification of the proposed method from a computational viewpoint in order to provide its advantages also for large scale systems.

\section{REFERENCES}

[1] N. Elia and S. K. Mitter. Stabilization of linear systems with limited information. IEEE Transactions on Automatic Control, 46(9):1384-1400, 2001.

[2] S. Tatikonda and S. K. Mitter. Control under communication constraints. IEEE Transactions on Automatic Control, 49(7):1056-1068, 2004.

[3] M. Fu and L. Xie. The sector bound approach to quantized feedback control. IEEE Transactions on Automatic Control, 50(11):1698-1711, 2005.

[4] A. Matveev and A. Savkin. Multirate stabilization of multiple sensor systems via limited capacity communication channels. SIAM Journal on Control and Optimization, 44(2):584-617, 2005.

[5] K. Li and J. Baillieul. Robust and efficient quantization and coding for control of multidimensional linear systems under data rate constraints. International Journal of Robust and Nonlinear Control, 17(10-11):898-920, 2007.

[6] S. Wan and L. Qiu. Stabilization of networked control systems with finite data rate. In Asian Control Conference, pages 1067-1073, Hong Kong, China, 2009.

[7] G. Stein. Respect the unstable. IEEE Control Systems Magazine, 23(4):12-25, 2003.

[8] G. Nair, R. J. Evans, I. Mareels, and W. Moran. Topological feedback entropy and nonlinear stabilization. IEEE Transactions on Automatic Control, 49(9):1585-1597, 2004.

[9] J. H. Braslavsky, R. H. Middleton, and J. S. Freudenberg. Feedback stabilization over signal-to-noise ratio constrained channels. IEEE Transactions on Automatic Control, 52(8):1391-1403, 2007.

[10] R. Bowen. Entropy for group endomorphisms and homogeneous space. Transactions of the American Mathematical Society, 153:401-414, 1971.

[11] L. Qiu. Quantify the unstable (semiplenary lecture). In International Symposium on Mathematical Theory of Networks and Systems, Budapest, Hungary, 2010.

[12] G. Chesi. Worst-case Mahler measure in polytopic uncertain systems. IEEE Transactions on Automatic Control, 57(12):3208-3213, 2012.

[13] G. Chesi. Instability analysis of uncertain systems via determinants and LMIs. IEEE Transactions on Automatic Control (to appear), 2015.

[14] G. Chesi, A. Garulli, A. Tesi, and A. Vicino. Characterizing the solution set of polynomial systems in terms of homogeneous forms: an LMI approach. International Journal of Robust and Nonlinear Control, 13(13):1239-1257, 2003.

[15] T. Mora. Solving Polynomial Equation Systems II. Cambridge University Press, 2005. 
[16] G. Chesi. On the unstable of continuous-time linearized nonlinear systems. In IEEE Conference on Decision and Control, pages 2316-2321, Los Angeles, California, 2014.

[17] R. Bellman. Introduction to Matrix Analysis. McGraw-Hill, New York, 1974.

[18] S. Boyd, L. El Ghaoui, E. Feron, and V. Balakrishnan. Linear Matrix Inequalities in System and Control Theory. SIAM, 1994.

[19] G. Chesi, A. Tesi, A. Vicino, and R. Genesio. On convexification of some minimum distance problems. In European Control Conference, Karlsruhe, Germany, 1999.

[20] M. Kojima. Sums of squares relaxations of polynomial semidefinite programs. Technical report, Tokyo Institute of Technology, 2003.

[21] S. Prajna, A. Papachristodoulou, and F. Wu. Nonlinear control synthesis by sum of squares optimization: a Lyapunov-based approach. In Asian Control Conference, 2004.

[22] C. W. J. Hol and C. W. Scherer. Computing optimal fixed order $H_{\infty}$-synthesis values by matrix sum of squares relaxations. In IEEE Conference on Decision and Control, pages 3147-3153, Paradise Island, Bahamas, 2004.

[23] G. Chesi. LMI techniques for optimization over polynomials in control: a survey. IEEE Transactions on Automatic Control, 55(11):2500-2510, 2010.

[24] G. Stengle. A nullstellensatz and a positivstellensatz in semialgebraic geometry. Math. Ann., 207:87-97, 1974.

[25] M. Putinar. Positive polynomials on compact semi-algebraic sets. Ind. Univ. Math., 42:969-984, 1993.

[26] J. F. Sturm. Using SeDuMi 1.02, a MATLAB toolbox for optimization over symmetric cones. Optimization Methods and Software, 11-12:625-653, 1999. 